\title{
JAK3 Inhibitor PF-06651600
}

\author{
National Cancer Institute
}

\section{Source}

National Cancer Institute. JAK3 Inhibitor PF-06651600. NCI Thesaurus. Code C161783.

An orally available small-molecule inhibitor of tyrosine-protein kinase JAK3 (Janus kinase 3; JAK3) with potential immunomodulatory and anti-inflammatory activities. Upon administration, JAK3 inhibitor PF-06651600 selectively and irreversibly binds to JAK3 and prevents the activation of the JAK-signal transducers and activators of transcription (STAT) signaling pathway. This may decrease the production of certain pro-inflammatory cytokines and prevent both an inflammatory response and the inflammation-induced damage caused by certain immunological diseases. By selectively targeting JAK3, PF06651600 may spare interleukin (IL)-6 signaling, as well as other JAK1-dependent immunoregulatory cytokines such as IL-10, IL-27, and IL-35, which may offer a safety benefit compared to non-selective JAK inhibitors. JAK kinases are intracellular enzymes involved in signaling pathways affecting hematopoiesis, immunity and inflammation. 